Tecnología y

Ciencias $\stackrel{\Xi}{\sim}$ ggua
2020, Instituto Mexicano de Tecnología del Agua

Open Access bajo la licencia CC BY-NC-SA 4.0

(https://creativecommons.org/licenses/by-nc-sa/4.0/)

DOI: $10.24850 /$ j-tyca-2020-02-07

Notas

\title{
Modelo de simulación para el drenaje agrícola subterráneo
}

\section{Simulation model for subsurface agricultural drainage}

Manuel Zavala ${ }^{1}$, ORCID: 0000-0002-0595-735X

Heber Saucedo ${ }^{2}$

Carlos Fuentes ${ }^{3}$

${ }^{1}$ Universidad Autónoma de Zacatecas, Zacatecas, Zacatecas, México, mzavala73@uaz.edu.mx

${ }^{2}$ Comisión Nacional de Agua, Ciudad de México, México, hebersaucedo@yahoo.com

${ }^{3}$ Instituto Mexicano de Tecnología del Agua, Jiutepec, Morelos, México, cfuentes@tlaloc.imta.mx

Autor para correspondencia: Manuel Zavala, mzavala73@uaz.edu.mx

\section{Resumen}

En este trabajo se presenta la segunda versión del programa de cómputo DRENAS (drenaje agrícola subterráneo), el cual incluye tres 
Tecnología y

Ciencias $₫$ Agua
2020, Instituto Mexicano de Tecnología del Agua

Open Access bajo la licencia CC BY-NC-SA 4.0

(https://creativecommons.org/licenses/by-nc-sa/4.0/)

soluciones analíticas y una solución de elemento finito tipo Galerkin de la ecuación diferencial del drenaje agrícola en su forma unidimensional; puede ejecutarse bajo sistemas operativos Windows de 32 bits y 64 bits. Los modelos analíticos y numéricos incluidos en la versión 1.0 del programa se reprogramaron en Visual Basic 2017, realizando modificaciones para mejorar sus capacidades de cálculo, registro de datos y visualización de resultados. El modelo numérico se amplió para resolver la ecuación de Boussinesq no lineal con coeficientes variables, incluyendo el término de recarga vertical, y para manejar las opciones de usar en la frontera de los drenes condiciones tipo Dirichlet, radiación lineal o radiación fractal. Los resultados proporcionados por el módulo de la solución numérica del programa fueron validados satisfactoriamente haciendo uso de una solución analítica y un experimento de drenaje.

Palabras clave: ecuación de Boussinesq, coeficiente de almacenamiento variable, condición de radiación fractal, propiedades hidráulicas del suelo.

\section{Abstract}

The second version of the computer program DRENAS (subsurface agricultural drainage) is presented, which includes four analytical solutions and a finite element solution Galerkin type of the differential equation of agricultural drainage in its one-dimensional form and can be run under Windows systems of 32-bit or 64-bit. The analytical and numerical models included in version 1.0 of the program were migrated from Visual Basic 2005 to Visual Basic 2017 and modified to expand and 
Tecnología y

Ciencias $₫$ Agua
2020, Instituto Mexicano de Tecnología del Agua

Open Access bajo la licencia CC BY-NC-SA 4.0

(https://creativecommons.org/licenses/by-nc-sa/4.0/)

improve their calculation, data recording and results visualization capabilities. The numerical model can now solve the non-linear Boussinesq equation with variable coefficients including the vertical recharge term and allows use at the boundary of the drains, Dirichlet condition, linear radiation or fractal radiation. The results provided by the module of the numerical solution of the program were validated using an analytical solution and a drainage test.

Keywords: Boussinesq equation, variable storage coefficient, fractal radiation condition, soil hydraulic properties.

Recibido: $14 / 03 / 2018$

Aceptado: 29/07/2019

\section{Introducción}

Los terrenos agrícolas que presentan problemas de salinidad y/o de manto freático somero pueden ser recuperados o controlados mediante drenaje artificial, que en el caso de la parcela puede ser del tipo subterráneo. La variables a determinar en el diseño de un sistema de drenaje subterráneo es la profundidad de los drenes, la separación de los mismos y su diámetro. El cálculo de estas variables debe considerar 
Tecnología y

Ciencias $₫$ Agua
2020, Instituto Mexicano de Tecnología del Agua

Open Access bajo la licencia CC BY-NC-SA 4.0

(https://creativecommons.org/licenses/by-nc-sa/4.0/)

las características del suelo (textura, estructura, propiedades físicas e hidráulicas) y la condición del flujo de agua en el subsuelo.

Es posible realizar estudios de los procesos de transferencia de agua en los sistemas de drenaje agrícola con la ecuación de Boussinesq de los acuíferos libres, que aunque considera de forma simplificada las transferencias de masa y energía en la zona vadosa del suelo, proporciona descripciones generales del flujo de agua en el espesor saturado del medio poroso (Ritzema, 2006). Para realizar modelaciones detalladas de la dinámica del agua con la ecuación de Boussinesq, en el caso de acuíferos libres someros se debe considerar la dependencia del coeficiente de almacenamiento del acuífero respecto de la carga hidráulica y usar la condición de frontera en los drenes que mejor represente el flujo de drenaje. Por una parte, Fuentes, Zavala \& Saucedo (2009) establecen formalmente la relación entre la curva de retención de humedad y el coeficiente de almacenamiento en acuíferos libres y, por otra, Zavala, Fuentes \& Saucedo (2007) demuestran que la transferencia de agua del suelo hacia el interior de los drenes debe ser descrita con una condición de radiación no lineal.

Zavala, Saucedo \& Fuentes (2014) desarrollan la primera versión computacional para aplicar la ecuación de Boussinesq con coeficiente de almacenamiento variable sujeta en los drenes a condiciones de radiación fractal, a la cual denominan DRENAS (drenaje agrícola subterráneo). Sin embargo, la herramienta de cómputo se programó en Visual Basic 2005, por lo que opera sólo en sistemas Windows de 32 bits, y sus opciones gráficas y base de datos dependen de Microsoft Office 2003. 
Tecnología y

Ciencias $₫$ Agua
2020, Instituto Mexicano de Tecnología del Agua

Open Access bajo la licencia CC BY-NC-SA 4.0

(https://creativecommons.org/licenses/by-nc-sa/4.0/)

El objetivo general de este trabajo fue desarrollar en Visual Basic 2017, la segunda versión del programa de cómputo DRENAS, para expandir sus capacidades de cálculo, mejorar su interfaz gráfica, y eliminar su dependencia de programas externos para el manejo de datos y elaboración de gráficos. En la actualización también se incorporaron y programaron nuevas opciones de cálculo, a fin de incrementar las alternativas de simulación, como el manejo del término de recarga o descarga de la ecuación de Boussinesq como una función dependiente del tiempo; representación de la condición inicial de la carga hidráulica como una función variable en el espacio; posibilidad de usar restricciones mecanicistas para los parámetros de forma de la curva de retención de humedad de Van Genuchten (1980) cuando se utiliza para definir el coeficiente de almacenamiento como función de la carga hidráulica, y la opción de usar condiciones de frontera en el dren del tipo Dirichlet. Con la meta de depurar el programa de cómputo, se realizó su validación, considerando una solución analítica para drenaje subterráneo en régimen estacionario y datos de una prueba de drenaje experimental de laboratorio.

\section{Materiales y métodos}

\section{Ecuaciones de base}


Tecnología y

Ciencias $\stackrel{\unlhd}{\unlhd}$ gua
2020, Instituto Mexicano de Tecnología del Agua

Open Access bajo la licencia CC BY-NC-SA 4.0

(https://creativecommons.org/licenses/by-nc-sa/4.0/)

En el programa de cómputo se resuelve la forma completa o simplificada de la ecuación de Boussinesq unidimensional del drenaje agrícola. En general, se puede escribir como:

$\mu(H) \frac{\partial H}{\partial t}=\frac{\partial}{\partial x}\left[T(H) \frac{\partial H}{\partial x}\right]+R_{w}(t)$

Donde $H=H(x, t)$ es la carga hidráulica contada a partir de un estrato impermeable o nivel de referencia (L) $y$ es función de la coordenada horizontal $x$ y del tiempo $t ; T(H)$ es la transmisibilidad del acuífero $\left(L^{2} T^{1}\right)$; para el caso de acuíferos libres es $T(H)=K_{s} H ; K_{s}$ es la conductividad hidráulica a saturación del suelo $\left(L T^{1}\right) ; \mu(H)$ es el coeficiente de almacenamiento del acuífero; $R_{w}(t)$ es el volumen de recarga o descarga en la unidad de tiempo por unidad de área del acuífero $\left(L T^{1}\right)$.

La recarga o descarga $R_{w}(t)$ es una variable difícil de determinar, ya que depende de las condiciones de flujo existentes en la superficie de suelo (lluvia, evaporación, tirante de agua), el flujo del agua y el régimen de humedad en la zona vadosa, la estratigrafía de la zona no saturada del suelo, la macroporosidad, grietas, etcétera. En esta nueva versión del modelo de simulación numérico se incorporó el término $R_{w}(t)$, representándolo mediante un polinomio que depende del tiempo: 
Tecnología y

Ciencias $\stackrel{\unlhd}{\unlhd}$ gua
2020, Instituto Mexicano de Tecnología del Agua

Open Access bajo la licencia CC BY-NC-SA 4.0

(https://creativecommons.org/licenses/by-nc-sa/4.0/)

$R_{w}(t>0)=a_{r} t^{3}+b_{r} t^{2}+c_{r} t+d_{r}$

Donde $a_{r}, b_{r}, c_{r}$ y $d_{r}$ son coeficientes a calcular, considerando mediciones experimentales o estimaciones que se tengan de la evolución en el tiempo de la recarga para un evento específico.

Se consideró en el programa la relación entre el coeficiente de almacenamiento y la curva de retención de humedad establecida por Fuentes et al. (2009):

$\mu(H)=\theta_{s}-\theta\left(H-H_{s}\right)$

Donde $\theta_{s}$ es el contenido volumétrico de agua a saturación $\left(L^{3} L^{-3}\right)$; y $H_{s}$ es la elevación de la superficie del suelo $(L)$. Es posible obtener una representación analítica explícita del coeficiente de almacenamiento a partir de la Ecuación (3) si la curva de retención de humedad del suelo es conocida. Se seleccionó la relación clásica de Van Genuchten (1980), ampliamente aceptada en estudios de campo y de laboratorio por su flexibilidad descriptiva:

$\theta(\psi)=\theta_{r}+\left(\theta_{s}-\theta_{r}\right)\left[1+\left(\psi / \psi_{d}\right)^{n}\right]^{-m}$

Donde $\theta_{r}$ es el contenido volumétrico residual $\left(L^{3} L^{-3}\right) ; \psi_{d}<0$ es un parámetro de escala del potencial de presión del agua en el suelo $\psi_{r}(L)$; $m$ y $n$ son parámetros de forma adimensionales. En esta nueva versión 
Tecnología y

Ciencias $\stackrel{\Xi}{\unlhd}$ gua
2020, Instituto Mexicano de Tecnología del Agua

Open Access bajo la licencia CC BY-NC-SA 4.0

(https://creativecommons.org/licenses/by-nc-sa/4.0/)

del programa de cómputo se incluyeron relaciones mecanicistas entre $m$ y $n$ que satisfacen la teoría de la infiltración, la restricción clásica de Burdine (1953) $m=1-2 / n$, así como las relaciones fractales de Fuentes, Brambila, Vauclin, Parlange \& Haverkamp (2001), que derivan en su estudio de la conductividad hidráulica de los suelos no saturados. Las restricciones fractales son las denominadas poro neutro $m=(1-$ $4 s / n) / s$, poro geométrico $m=(1-2 s / n) / s$ y poro grande $m=(1-$ $4 s / n) / 2 s$; donde $s$ es la dimensión cociente del objeto fractal, tal como se considera el suelo en ese estudio, siendo $s$ la razón entre la dimensión fractal del objeto $D_{f}$ (Mandelbrot, 1982; Falconer, 2014) y la dimensión del espacio de Euclides $E, s=D_{f} / E$.

Al introducir la Ecuación (4) en la Ecuación (3) se obtuvo la siguiente relación:

$$
\mu(H)=\left(\theta_{s}-\theta_{r}\right)\left\{1-\left[1+\left\{\left(H-H_{s}\right) / \psi_{d}\right\}^{n}\right]^{-m}\right\}
$$

Se retuvo en esta segunda versión del programa la condición de radiación fractal para los drenes derivada por Zavala et al. (2007):

$q_{d}=\gamma K_{\text {in }}\left[\left(H_{d}-D_{o}\right) / P\right]^{2 \bar{s}}$

Donde $q_{d}$ es el flujo de drenaje; $\gamma$ es un coeficiente de conductancia adimensional; $K_{i n}=\sqrt{ }\left(K_{s} K_{d}\right)$ es la conductividad de la interfaz suelo-dren; $K_{s}$ es la conductividad hidráulica a saturación del suelo; $K_{d}$ la conductividad de la pared del dren; $P$, la profundidad de los Tecnología y ciencias del agua, ISSN 2007-2422, 11(2), 262-290. DOI: 10.24850/j-tyca-2020-02-07 
Tecnología y

Ciencias $₫$ Agua
2020, Instituto Mexicano de Tecnología del Agua

Open Access bajo la licencia CC BY-NC-SA 4.0

(https://creativecommons.org/licenses/by-nc-sa/4.0/)

drenes; $H_{d}$, la carga hidráulica en la posición del dren; $D_{o}$, el espesor del acuífero; $\bar{s}=(1 / 2)\left(s_{1}+s_{d}\right)$ es la dimensión cociente en la interfaz suelodren; $s_{1}$ es la dimensión cociente del suelo y $s_{d}$ es la dimensión cociente de la pared del dren. La relación entre la porosidad areal $\left(\mu_{\text {areal }}\right)$ y la porosidad volumétrica $(\phi)$ se obtiene de acuerdo con la idea probabilista como $\mu_{\text {areal }}=\phi^{s} \phi^{s}=\phi^{2 s}$, se tiene que la porosidad volumétrica:

$$
(1-\phi)^{s}+\phi^{2 s}=1
$$

y la porosidad areal:

$$
\left(1-\mu_{\text {areal }}\right)^{\frac{1}{s}}+\mu_{\text {areal }}^{\frac{1}{2 s}}=1
$$

La conductividad de la pared del dren se obtiene con una fórmula basada en la ley de Poiseuille: $K_{d}=(1 / 2)(g / v) \mu_{\text {areal_}} d\left(R_{H D}\right)^{2}$, donde $g$ es la aceleración de la gravedad; $v$, la viscosidad cinemática del agua; $R_{H D}$, el radio hidráulico del dren. Se introdujo en el versión 2.0 de DRENAS la opción de manejar directamente la condición de radiación lineal $(\bar{s}=0.5$ en la Ecuación (6)) sin necesidad de utilizar las rutinas de cálculo de $s_{1}$ y $S_{d}$.

Modelaciones numéricas simplificadas del drenaje agrícola se pueden llevar a cabo imponiendo en los drenes condiciones de frontera tipo Dirichlet (carga hidráulica en la posición espacial del dren). En la nueva versión del programa de cómputo se incorporó esta condición de 
Tecnología y

Ciencias $\stackrel{\Xi}{\unlhd}$ gua
2020, Instituto Mexicano de Tecnología del Agua

Open Access bajo la licencia CC BY-NC-SA 4.0

(https://creativecommons.org/licenses/by-nc-sa/4.0/)

frontera como una alternativa a la condición de radiación (6); se seleccionó la siguiente función, que depende de la variable independiente tiempo:

$H\left(x_{d r e n}, t>0\right)=a_{d} t+b_{d} t^{\frac{1}{2}}+c_{d}+d_{d} t^{-\frac{1}{2}}$

Donde $x_{d r e n}$ es la coordenada horizontal donde se ubica el dren; y $a_{d}, b_{d}, c_{d}$ y $d_{d}$ son coeficientes que se deben calcular a partir de mediciones que se tengan de la evolución de la carga hidráulica sobre el dren. La Ecuación (9) tiene la flexibilidad de describir comportamientos extremos de la carga sobre el dren, como el abatimiento y ascenso.

Por último, para realizar la simulación numérica con la Ecuación (1) debe definirse el estado inicial de la carga hidráulica en el sistema. En este trabajo se incluyó la opción de usar hasta un polinomio de tercer grado, que es función de la coordenada horizontal $x$ :

$H(x, t=0)=a_{p} x^{3}+b_{p} x^{2}+c_{p} x+d_{p}$

Donde $a_{p}, b_{p}, c_{p}$ y $d_{p}$ son coeficientes a determinar a partir de mediciones de la carga hidráulica en el sistema en el instante inicial de cálculo o simulación.

En la solución numérica del sistema (1)-(10) se empleó el método del elemento finito tipo Galerkin para la discretización espacial; un esquema de diferencias finitas para la discretización temporal; el 
Tecnología y

Ciencias $\stackrel{\Xi}{\unlhd}$ gua
2020, Instituto Mexicano de Tecnología del Agua

Open Access bajo la licencia CC BY-NC-SA 4.0

(https://creativecommons.org/licenses/by-nc-sa/4.0/)

método iterativo de Picard para la linealización del sistema resultante, y un método de gradiente conjugado precondicionado de para la solución del sistema de ecuaciones algebraicas (Zavala et al., 2014; Zienkiewicz, Taylor, \& Zhu, 2013; Noor \& Peters, 1987). El sistema de ecuaciones resultante de la discretización se programó en Visual Basic 2017.

Es conocido que cálculos simplificados de separación entre drenes y abatimiento del manto freático pueden ser realizados aplicando soluciones analíticas obtenidas para formas reducidas de la ecuación de Boussinesq (Ecuación (1)); por ejemplo, para régimen de flujo permanente se tiene la fórmula de Hooghoudt (1940), y en régimen transitorio las relaciones de Glover-Dumm (Dumm, 1954) y Fuentes et al. (1997), las cuales ya se incluían en la versión original del programa de cómputo y se retienen en la actualización.

\section{Desarrollo de la interfaz gráfica}

En la segunda versión del programa de cómputo DRENAS, se reprogramaron en Visual Basic 2017 todos los módulos de captura de información de la versión original; se desarrollaron bases de datos internas independientes de Microsoft Access; se desvinculó de Microsoft Excel el tratamiento de las gráficas, y se generó el archivo ejecutable para instalar DRENAS 2.0 en cualquier computadora que disponga de 
Tecnología y

Ciencias $\stackrel{\Xi}{\unlhd}$ gua
2020, Instituto Mexicano de Tecnología del Agua

Open Access bajo la licencia CC BY-NC-SA 4.0

(https://creativecommons.org/licenses/by-nc-sa/4.0/)

sistema operativo Windows de 64 bits, e incluso se generó también la opción para sistemas Windows 32 bits.

El programa DRENAS 2.0 tiene dos módulos de cálculo: uno denominado soluciones analíticas y otro modelo numérico (Figura 1). El módulo soluciones analíticas tiene cuatro secciones: dos para realizar cálculos para condiciones de flujo de agua en régimen permanente usando la solución de Hooghoudt (1940) y dos para condiciones de flujo en régimen transitorio; uno para realizar cálculos con la solución de Dumm (1954) y el otro con la solución de Fuentes et al. (1997). En estas cuatro secciones se puede calcular la separación entre drenes o el módulo de drenaje. Respecto a la versión original del modelo DRENAS, en la versión 2.0 estas secciones fueron mejoradas, al incorporar las siguientes opciones: a) almacenamiento de las simulaciones realizadas en una base de datos interna; b) carga de ejemplos previos; c) generación de un reporte de la simulación y alternativa para exportarlo a un archivo pdf; d) La gráfica de resultados también puede ser exportada como imagen. Un ejemplo de una sección de cálculo del módulo de soluciones analíticas se presenta en la Figura 2. 
2020, Instituto Mexicano de Tecnología del Agua

Tecnología y

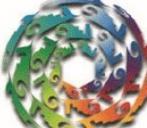

Ciencias ฐ̊Agua

(https://creativecommons.org/licenses/by-nc-sa/4.0/)

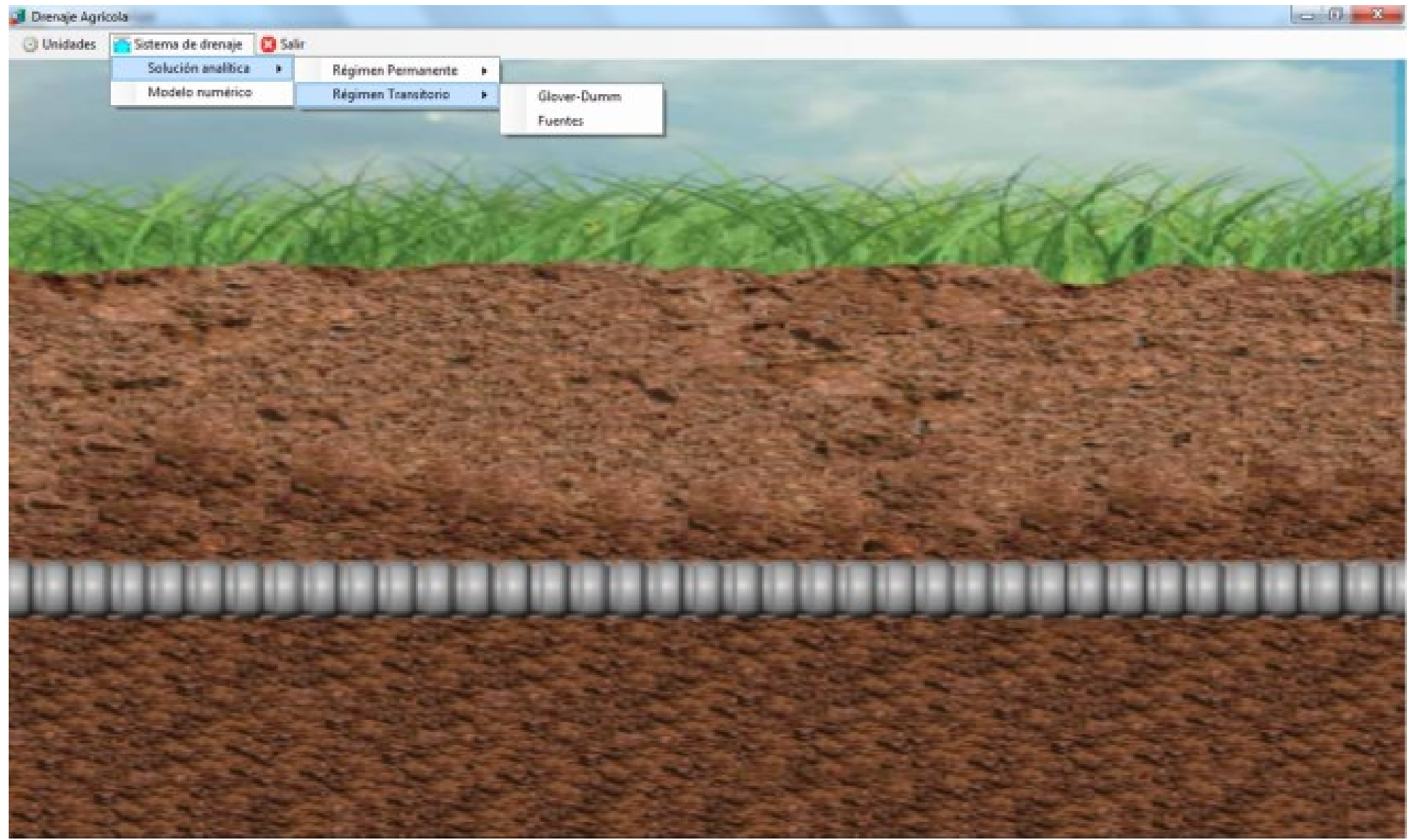

Figura 1. Pantalla general del programa DRENAS 2.0. 


\section{0, Instituto Mexicano de Tecnología del Agua}

Ciencias $₫$ Agua

Open Access bajo la licencia CC BY-NC-SA 4.0

(https://creativecommons.org/licenses/by-nc-sa/4.0/)

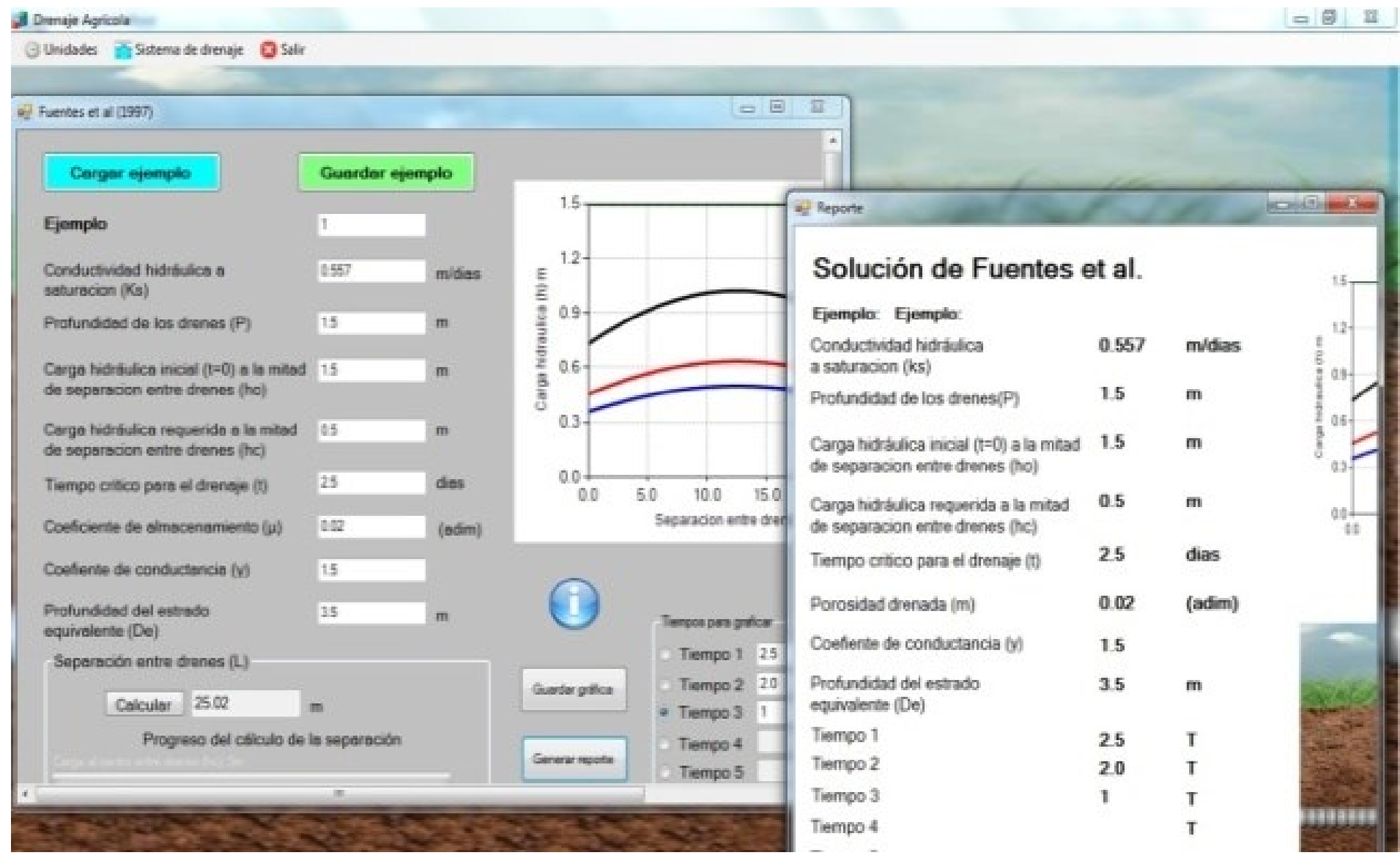

Figura 2. Una sección de cálculo del módulo soluciones analíticas.

El módulo de la solución numérica se programó en una forma Tab Index (en inglés), que contiene seis pestañas o secciones: las primeras cuatro están desarrolladas para la captura de datos referente al sistema de drenaje, características del dren, propiedades del suelo y datos necesarios para la simulación numérica, como son los relacionados con el control de tiempo, discretización espacial, condiciones límite y visualización gráfica de resultados; la quinta pestaña sirve para almacenar los datos del ejemplo en una base de datos interna, y la última pestaña contiene el botón para ejecutar la simulación numérica. 
Tecnología y

Ciencias $\stackrel{\unlhd}{\unlhd}$ gua
2020, Instituto Mexicano de Tecnología del Agua

Open Access bajo la licencia CC BY-NC-SA 4.0

(https://creativecommons.org/licenses/by-nc-sa/4.0/)

Las nuevas capacidades del módulo de simulación numérica de la versión 2.0 de DRENAS que se programaron se establecieron de la siguiente forma:

a) Primero de la base de datos de suelos UNSODA 2.0 (Nemes, Schaap, Leij, \& Wösten, 2001) se seleccionaron las curvas experimentales de retención de humedad de 208 suelos reportados en la literatura; se ajustaron con la Ecuación (4), considerando las restricciones mecanicistas entre $m$ y $n$ antes descritas (Zavala, Saucedo, \& Fuentes, 2018), y los resultados obtenidos se incluyeron en una base de datos interna del programa de cómputo para ponerlos a la disposición de los usuarios del mismo (Figura 3). Si se selecciona la opción de usar datos de esta base del programa, al elegir un suelo, las secciones de propiedades físicas, hidráulicas y coeficiente de almacenamiento se llenan automáticamente con sus parámetros correspondientes. La mejora a resaltar en esta nueva versión es que se pueden manejar las relaciones entre $m$ y $n$ de los modelos de Burdine, poro neutro, poro geométrico y poro grande (Figura 4). 
Tecnología y

\section{Ciencias $₫$ Agua}

2020, Instituto Mexicano de Tecnología del Agua

Open Access bajo la licencia CC BY-NC-SA 4.0

(https://creativecommons.org/licenses/by-nc-sa/4.0/)

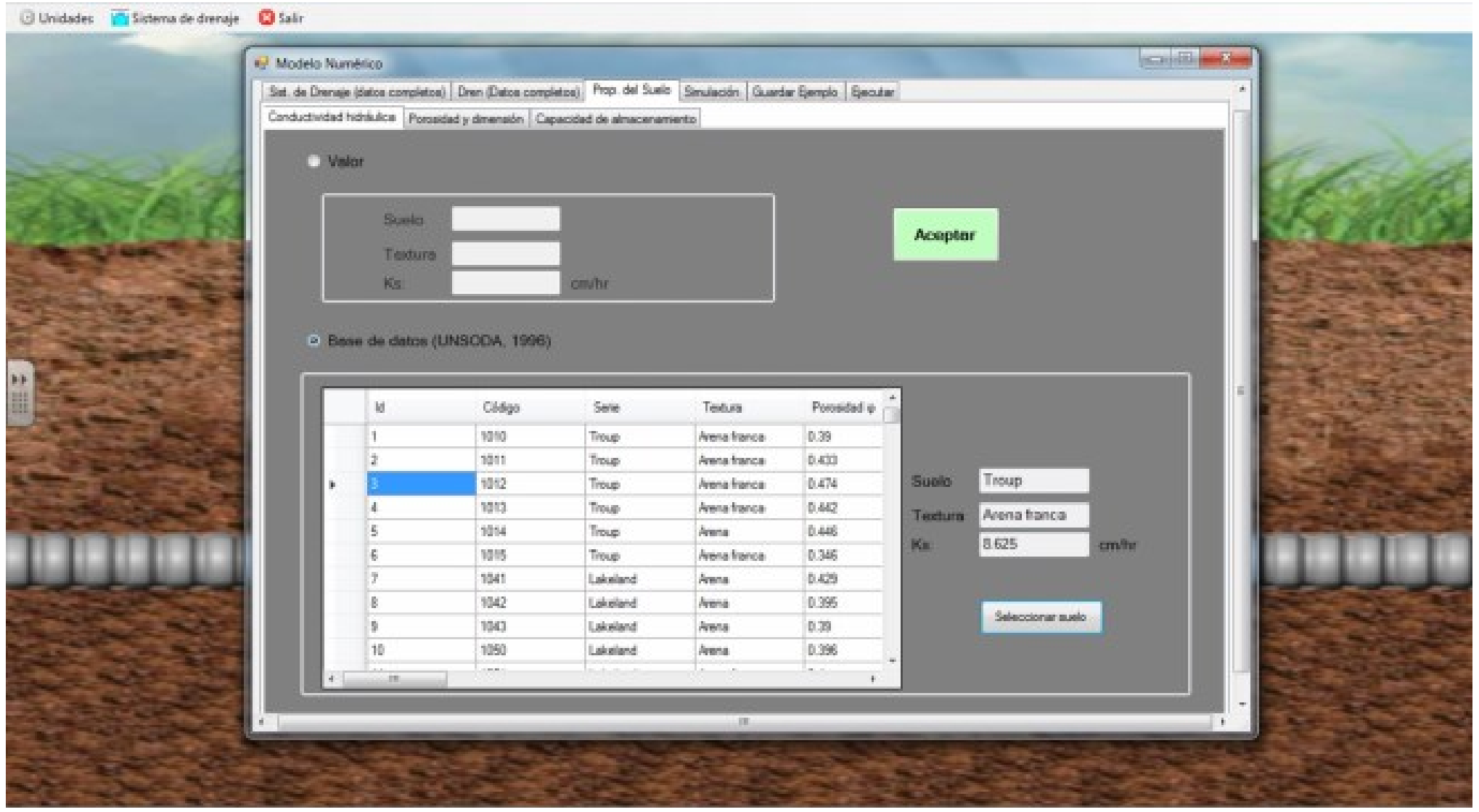

Figura 3. Módulo numérico: base UNSODA ampliada. 
Tecnología y

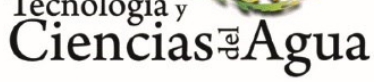

2020, Instituto Mexicano de Tecnología del Agua

Open Access bajo la licencia CC BY-NC-SA 4.0

(https://creativecommons.org/licenses/by-nc-sa/4.0/)

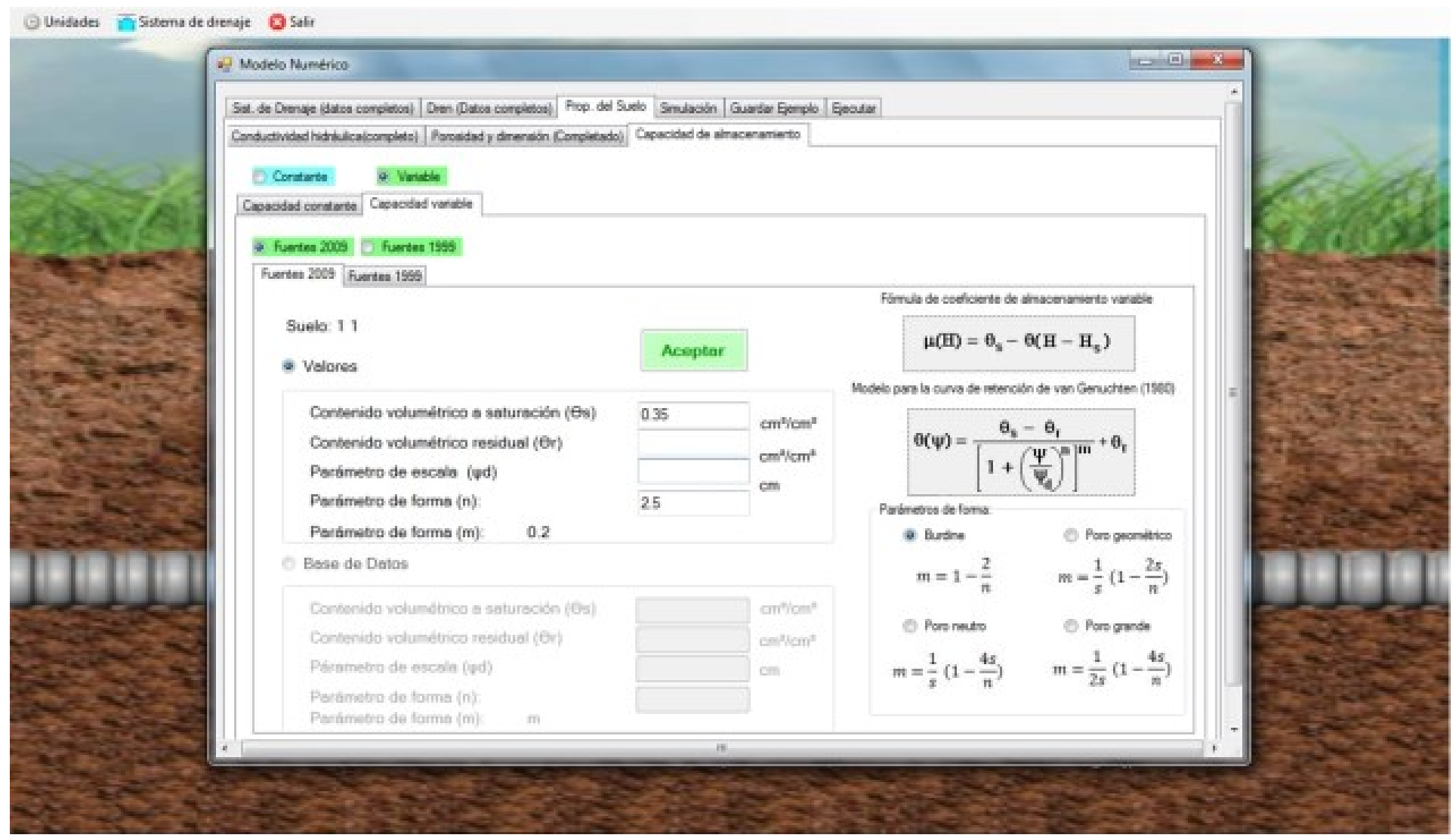

Figura 4. Módulo numérico: propiedades suelo-agua.

b) En la solución numérica de la ecuación de Boussinesq se incorporó el término de recarga $R_{w}(t)$ como una función del tiempo (Ecuación (2)); se realizaron las modificaciones de programación necesarias para poder imponer en los drenes la condición de frontera tipo Dirichlet (Ecuación (9)), y se programó también la condición inicial tipo polinomio cúbico (Ecuación (10)). En la Figura 5 se presenta la nueva sección desarrollada para incluir estas tres condiciones. 
Tecnología y

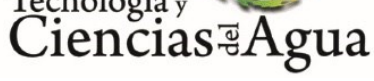

2020, Instituto Mexicano de Tecnología del Agua

Open Access bajo la licencia CC BY-NC-SA 4.0

(https://creativecommons.org/licenses/by-nc-sa/4.0/)

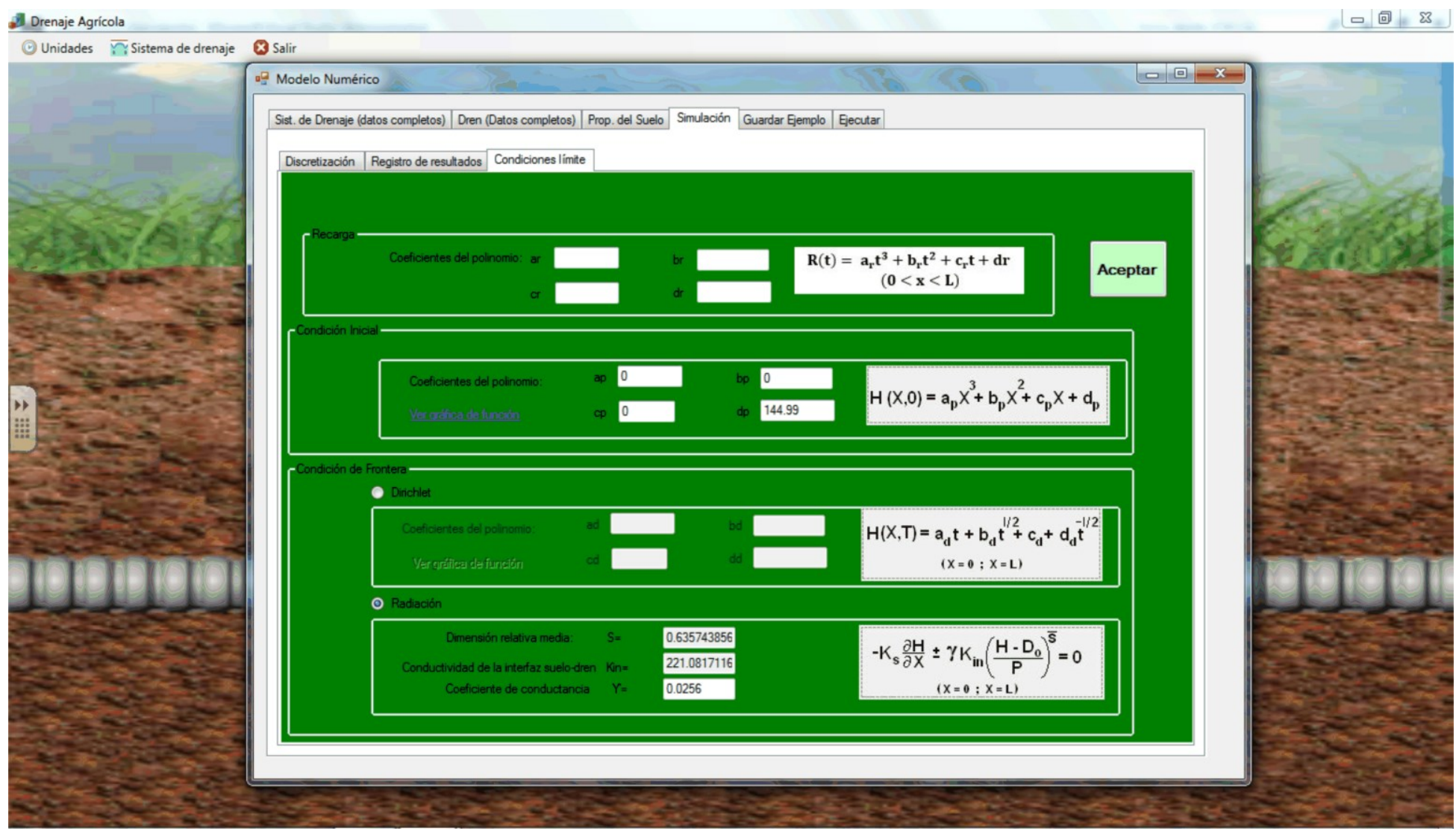

Figura 5. Módulo numérico: condiciones límite y recarga.

c) Se desarrolló una nueva sección para registrar los datos de la simulación, cargar datos para comparar simulaciones y para seleccionar el tipo de gráfica a visualizar en tiempo real que no depende de programas externos (Figura 6). 


\section{0, Instituto Mexicano de Tecnología del Agua}

Tecnología y

Ciencias $₫$ Agua
Open Access bajo la licencia CC BY-NC-SA 4.0

(https://creativecommons.org/licenses/by-nc-sa/4.0/)

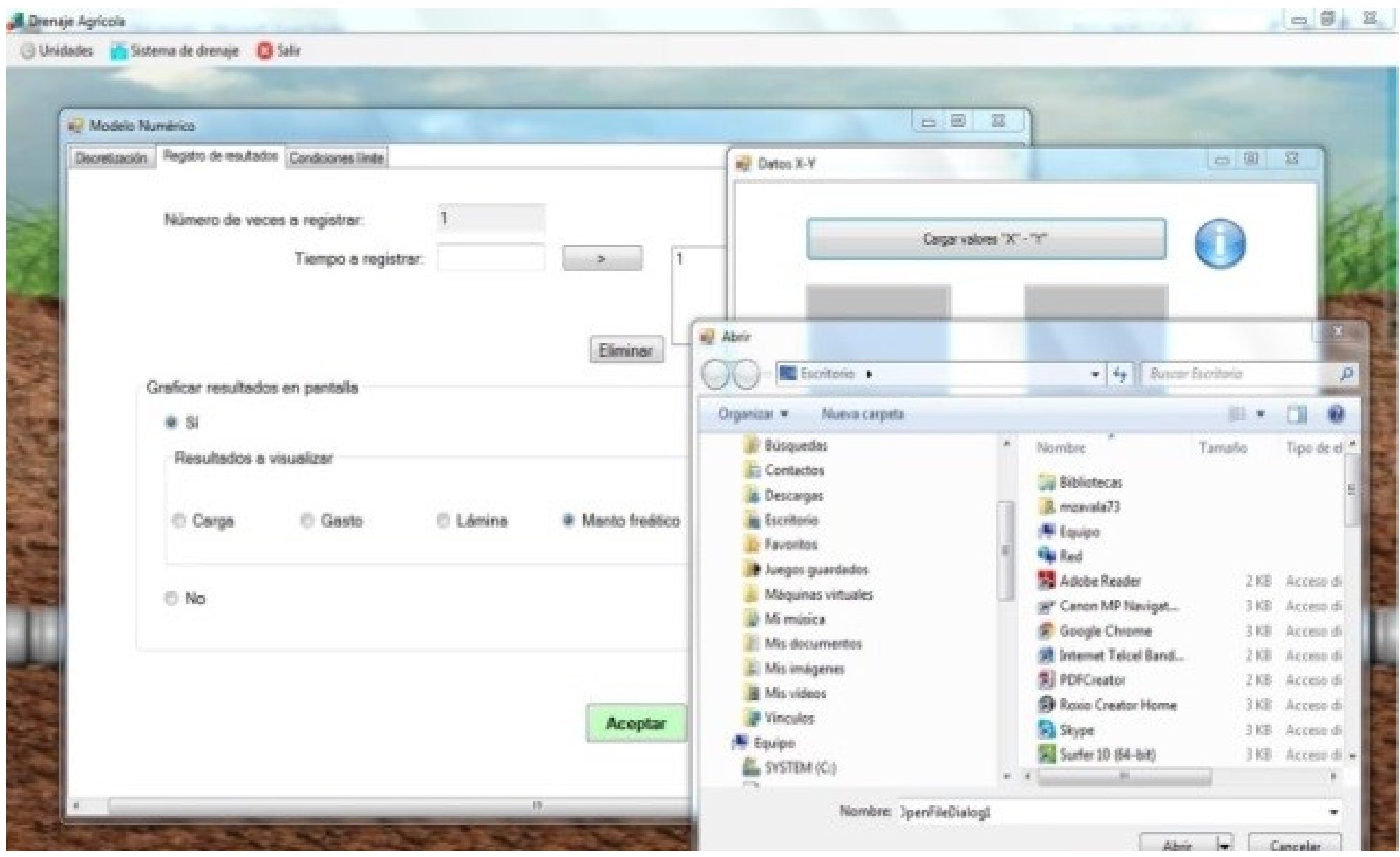

Figura 6. Módulo numérico: gráficas de la simulación.

\section{Resultados y discusión}


Tecnología y

Ciencias $₫$ Agua
2020, Instituto Mexicano de Tecnología del Agua

Open Access bajo la licencia CC BY-NC-SA 4.0

(https://creativecommons.org/licenses/by-nc-sa/4.0/)

Los resultados del programa de cómputo se compararon contra resultados externos (solución analítica y una prueba de drenaje en laboratorio) para detectar y corregir errores de programación, así como revisar la consistencia de las soluciones codificadas.

\section{Validación 1}

Se seleccionó la solución analítica del tipo Hooghoudt presentada en Fragoza et al. (2003), que considera radiación lineal $(\bar{s}=0.5$ en la Ecuación (6)) en los drenes para describir la evolución de la superficie libre en un sistema de drenaje subterráneo con recarga constante $\left(R_{0}\right)$ y flujo de agua en régimen permanente:

$H^{2}(x)=H_{c}^{2}+\left(R_{o} / K_{s}\right) x(L-x)$

donde $H_{c}$ es la carga hidráulica al centro entre drenes. Si se define $h(x, t)=H(x, t)-D_{o}$ y considera la condición de radiación lineal se tiene:

$h_{d}=\left[\sqrt{(4+\gamma)^{2} D_{o}^{2}+8(2+\gamma) h_{c}\left(h_{c}+2 D_{o}\right)}-(4+\gamma) D_{o}\right] /[2(2+\gamma)]$ 
Tecnología y

Ciencias $₫$ Agua
2020, Instituto Mexicano de Tecnología del Agua

Open Access bajo la licencia CC BY-NC-SA 4.0

(https://creativecommons.org/licenses/by-nc-sa/4.0/)

donde $h_{d}$ es la carga hidráulica sobre el dren $(x=0$ y $x=L) ; D_{o}$ es el espesor del acuífero; y $\gamma$ el coeficiente de conductancia de la condición de radiación lineal que se usa en los drenes. Analizando la Ecuación (2) se tiene para este caso que $a_{r}=b_{r}=c_{r}=0$ y $d_{r}=R_{o}$.

Los datos del sistema de drenaje son los reportados por Fragoza et al. (2003), derivados de un experimento de campo realizado en el distrito de riego 076 Valle del Carrizo, Sinaloa. El sistema de drenaje evaluado tiene una separación entre drenes $L=50 \mathrm{~m}$, profundidad media de los drenes $P=1.5 \mathrm{~m}$, profundidad del estrato impermeable $D_{0}$ $=3.5 \mathrm{~m}$, elevaciones del estrato impermeable y de la superficie del suelo $H_{i}=0.0 \mathrm{~m}$ y $H_{s}=5.0 \mathrm{~m}$. La parcela tiene suelo de textura arcillosa, con una conductividad hidráulica a saturación de $K_{s}=0.557 \mathrm{~m} / \mathrm{d}$, y el coeficiente de almacenamiento $\mu=0.1087$. Se asume $\gamma=1.5$ y $h_{c}=0.5$ $\mathrm{m}$. Con la Ecuación (12) se tiene $h_{d}=0.365 \mathrm{~m}$ y el valor de recarga obtenido con la condición para régimen permanente donde la descarga por el dren es igual a la recarga, $R_{o}=0.000944 \mathrm{~m} / \mathrm{d}$. Con estos valores se aplicó la Ecuación (11) para obtener la distribución espacial de la carga hidráulica a lo largo de la separación entre drenes.

Se reprodujo con el programa de cómputo el escenario descrito, modelándose el proceso transitorio flujo de agua en el sistema hasta alcanzar el régimen estacionario, el cual corresponde al descrito con la solución analítica. Se asumió la condición inicial de carga hidráulica constante $H(x, t=0)=4.50 \mathrm{~m}$ (por tanto, $a_{p}=b_{p}=c_{p}=0$ y $d_{p}=4.50 \mathrm{~m}$ en la Ecuación (10)) y para tener las mismas condiciones de la solución analítica, en el programa se usó la condición de frontera de radiación lineal, $\bar{s}=0.5$ en la Ecuación (6). Se consideró la hipótesis de igualdad 
Tecnología y

Ciencias $\stackrel{\Xi}{\triangleleft}$ Aua
2020, Instituto Mexicano de Tecnología del Agua

Open Access bajo la licencia CC BY-NC-SA 4.0

(https://creativecommons.org/licenses/by-nc-sa/4.0/)

entre la conductividad del suelo y la de la pared del dren $K_{s}=K_{d}$, lo cual genera $K_{\text {in }}=K_{s}=K_{d}$. Se reinterpretó el valor del coeficiente de conductancia reportado por Fragoza et al. (2003), para adaptarlo a la forma de radiación que se maneja en DRENAS 2.0, obteniéndose $\gamma=$ 0.045. La discretización espacial se realizó con el número de nodos $N=$ $1000(\Delta x=0.05 \mathrm{~m})$, y en la discretización del tiempo se usó $\Delta t_{i n i}=$ $1.157 \mathrm{E}-05 \mathrm{~d}, \Delta t_{\max }=6.94 \mathrm{E}-04 \mathrm{~d}$, y $\Delta t_{\min }=1.157 \mathrm{E}-06 \mathrm{~d}$. El tiempo de simulación fue de 720 días.

En la Figura 7 ( $\mathrm{a}$ y b) se presentan los resultados de la evolución del manto freático a la semana y al año de drenaje libre. Se observa que la solución numérica tiende a la solución analítica conforme avanza el tiempo y la reproduce de forma correcta. Esta comparación es una evidencia de que los módulos de captura de información de la solución numérica del programa y en particular el módulo de cálculo que maneja el término de la recarga vertical, condición inicial y condición de frontera, operan bien y están libres de errores de programación; también se observa que las series simuladas son estables y libres de oscilaciones numéricas. 
2020, Instituto Mexicano de Tecnología del Agua

Open Access bajo la licencia CC BY-NC-SA 4.0

(https://creativecommons.org/licenses/by-nc-sa/4.0/)

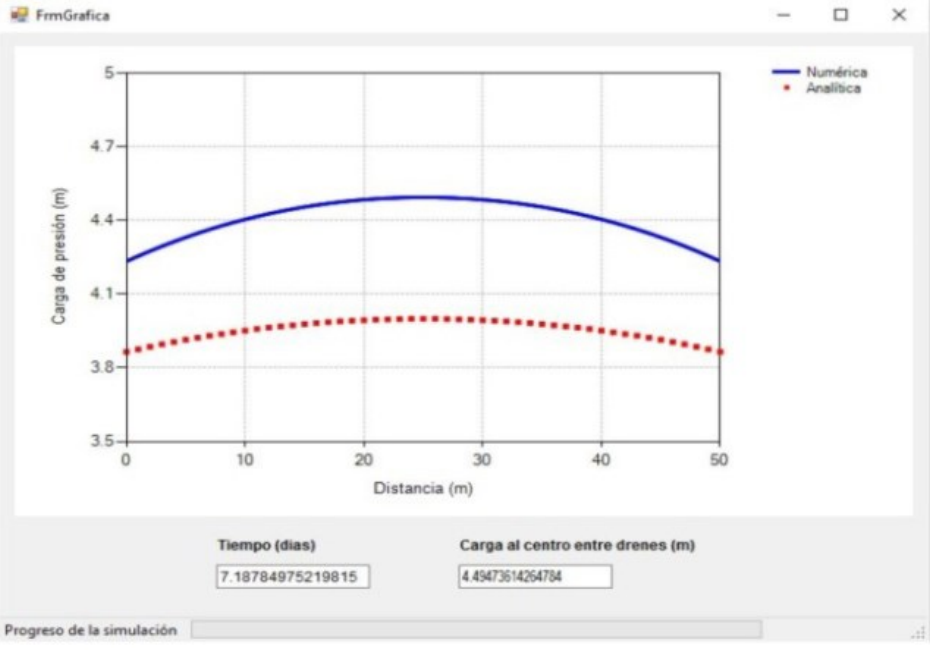

a) Primera semana de drenaje

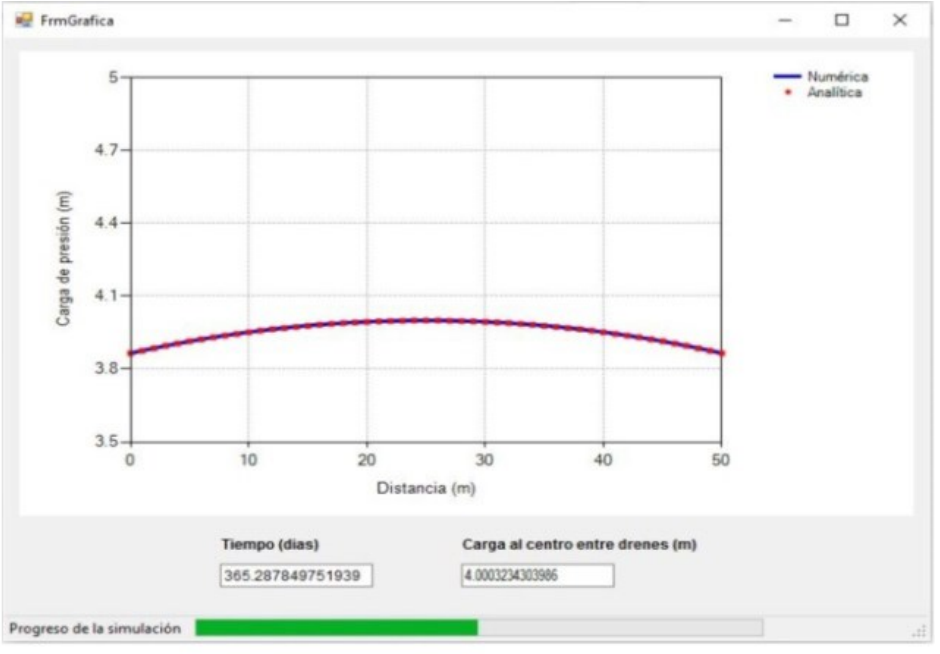

b) Un año de drenaje

Figura 7. Simulación de un escenario de drenaje agrícola (coeficiente de almacenamiento constante y recarga vertical constante).

\section{Validación 2}

Zavala, Fuentes \& Saucedo (2004) realizaron un experimento en un módulo de drenaje en el que se instalaron dos drenes de $30 \mathrm{~cm}$ de longitud $\left(I_{D}\right)$ y $5 \mathrm{~cm}$ de diámetro $\left(D_{D}\right)$, con $N_{o}=233$ perforaciones circulares de diámetro $d_{o}=0.158 \mathrm{~cm}$, distribuidas de modo uniforme en la superficie de cada tubo. Las dimensiones de cada dren: $L=100 \mathrm{~cm}, P$ $=120 \mathrm{~cm}$ y $D_{o}=25 \mathrm{~cm}$. El módulo se rellenó con suelo de textura arenosa, que se saturó aplicando una carga de agua constante en la 
Tecnología y

Ciencias $\stackrel{\unlhd}{\unlhd}$ gua
2020, Instituto Mexicano de Tecnología del Agua

Open Access bajo la licencia CC BY-NC-SA 4.0

(https://creativecommons.org/licenses/by-nc-sa/4.0/)

superficie del suelo hasta eliminar el aire. Con los drenes tapados se removió el exceso de agua en la superficie y se cubrió para eliminar la evaporación. Por último, se destaparon los drenes y se midió durante 10 días (240 horas) el volumen de agua drenado; se debe notar que la condición inicial corresponde a $H(x, 0)=P+D_{o}\left(d_{p}=145 \mathrm{~cm}\right.$ en la Ecuación (10) y el resto de sus coeficientes son nulos) y la recarga es nula $\left(R_{w}=0\right.$ en la Ecuación (2)).

La porosidad del suelo fue $\Phi_{1}=0.5396 \mathrm{~cm}^{3} / \mathrm{cm}^{3}$, y con este valor se resolvió la Ecuación (7), obteniéndose $s_{1}=0.7026$. El valor de la conductividad hidráulica a saturación reportado es $K_{s}=18.3 \mathrm{~cm} / \mathrm{h}$. El coeficiente de almacenamiento del acuífero descrito con la Ecuación (5) para los parámetros $\theta_{s}=\Phi_{1}, \theta_{r}=0, \Psi_{d}=-41.8 \mathrm{~cm}$ y $n=2 /(1-m)=$ 3.19 (restricción de Burdine, 1953).

La porosidad areal del dren es $\mu_{\text {areal } \_d}=A_{o} / A_{d}=0.0097 \mathrm{~cm}^{2} / \mathrm{cm}^{2}$ y al resolver la Relación (8) se tiene $s_{d}=0.5688$. Con el radio hidráulico de los orificios circulares de la pared del dren $R_{H D}=0.0395 \mathrm{~cm}$, la viscosidad cinemática del agua $v=36 \mathrm{~cm}^{2} / \mathrm{h}$ se aplica la ley de Poiseuille para calcular la conductividad de la pared del dren $\mathrm{K}_{d}=$ $2,670.8 \mathrm{~cm} / \mathrm{h}$. Los valores de la conductividad en la interfaz suelo-dren y la dimensión relativa son $\mathrm{K}_{\text {in }}=221.08 \mathrm{~cm} / \mathrm{h}$ y $\bar{s}=0.6357$. El coeficiente $\gamma$ de la radiación fractal reportado por Zavala et al. (2004) se reinterpretó para adaptarlo a la forma de radiación que se maneja en DRENAS 2.0 (Ecuación (6)), obteniéndose $\gamma=0.0749$.

El coeficiente de almacenamiento variable y la condición inicial de saturación total del medio poroso originan que la simulación deba ser ejecutada empleando pasos de tiempo pequeños al inicio de la misma, 
Teçnología y

Ciencias $₫$ Agua
2020, Instituto Mexicano de Tecnología del Agua

Open Access bajo la licencia CC BY-NC-SA 4.0

(https://creativecommons.org/licenses/by-nc-sa/4.0/)

lo cual minimiza problemas de convergencia y estabilidad numérica. Los pasos de tiempo utilizados son $\Delta t_{i n i}=2.77 \mathrm{E}-04 \mathrm{~h}, \Delta t_{i n i}=2.77 \mathrm{E}-05 \mathrm{~h}$ y $\Delta t_{\max }=0.2 \mathrm{~h}$. El número de nodos para discretizar el espacio es de 200.

En la Figura 8 ( $a$ y b) se presenta, respectivamente, el proceso de captura de datos de la simulación y la evolución de la lámina drenada. En la Figura $8 \mathrm{~b}$ se puede observar que el programa de cómputo reproduce de manera satisfactoria los datos experimentales de lámina drenada, teniéndose una solución numérica estable y libre de oscilaciones, lo cual es un indicador de que el módulo de coeficiente de almacenamiento variable trabaja de forma correcta. Los pasos de tiempo y espacio utilizados en la simulación son adecuados, ya que la lámina drenada total calculada $(23.915 \mathrm{~cm})$ difiere de la lámina medida (23.92 cm) en sólo 0.03\%.

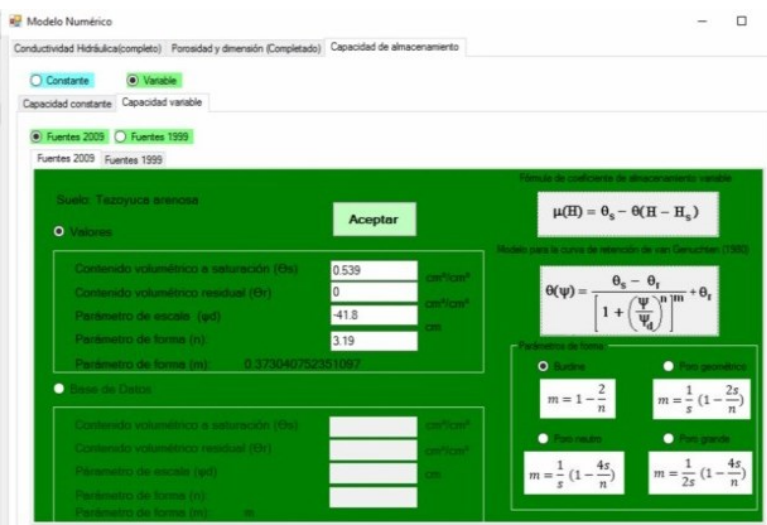

a) Captura de información: coeficiente de almacenamiento variable

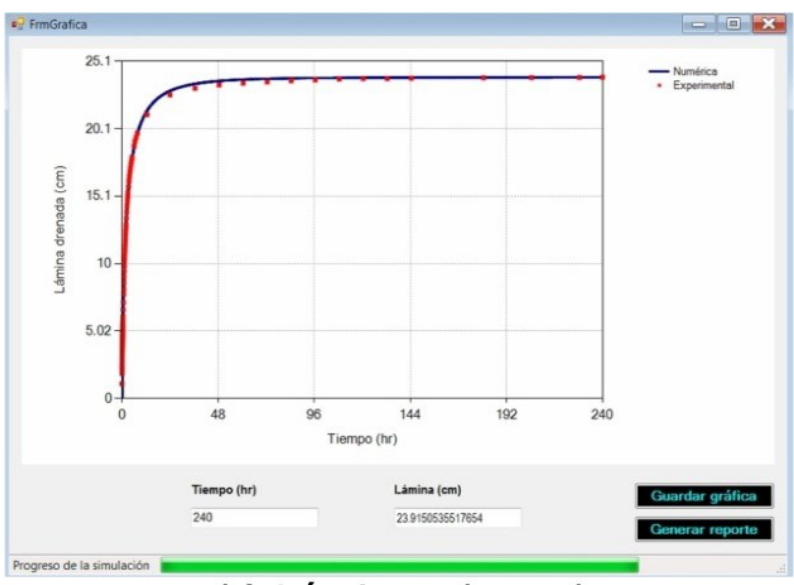

b) Láminas drenada experimental y simulada

Figura 8. Simulación de un escenario de drenaje agrícola (coeficiente de almacenamiento variable, recarga nula y condición de radiación fractal en los drenes). 
Tecnología y

Ciencias $\stackrel{\Xi}{\approx}$ Aua
2020, Instituto Mexicano de Tecnología del Agua

Open Access bajo la licencia CC BY-NC-SA 4.0

(https://creativecommons.org/licenses/by-nc-sa/4.0/)

\section{Conclusiones}

La nueva versión del programa de cómputo DRENAS (versión 2.0) es útil para describir el funcionamiento hidráulico de drenes subterráneos, para diseñar un sistema de drenaje y resolver problemas inversos de caracterización hidrodinámica de suelos a partir de eventos de drenaje. Esta versión de la herramienta computacional incorpora las siguientes funciones: a) bases internas para almacenar los datos ingresados en cada uno de los ejemplos que se analizan; b) base de suelos UNSODA, ampliada con parámetros para los modelos de conductividad hidráulica y retención de humedad del poro neutro, poro de la media geométrica y poro grande; c) opción para representar la recarga o descarga del acuífero como una función del tiempo; d) opción para representar la condición inicial de la carga hidráulica como función del espacio y usar condiciones tipo Dirichlet en los drenes variables en el tiempo; e) genera reportes de las simulaciones y permite exportar las gráficas de resultados. La visualización gráfica de las modelaciones evita la dependencia de programas externos, y se ejecuta sobre sistemas operativos Windows de 32 y 64 bits.

\section{Referencias}


Tecnología y

Ciencias $\stackrel{\unlhd}{\text { Agua }}$
2020, Instituto Mexicano de Tecnología del Agua

Open Access bajo la licencia CC BY-NC-SA 4.0

(https://creativecommons.org/licenses/by-nc-sa/4.0/)

Burdine, N. T. (1953). Relative permeability calculation from size distribution data. Petroleum transactions, AIME, 198, 71-78.

Dumm, L. D. (1954). New formula for determining depth and spacing of subsurface drains in irrigated lands. Agricultural Engineering, 35, 726-730.

Falconer, K. (2014). Fractal geometry: Mathematical foundations and applications. New York, USA: Wiley, John Wiley \& Sons, Ltd.

Fragoza, F., Fuentes, C., Zavala, M., Zatarain, F., Saucedo, H., \& Mejía, E. (2003). Drenaje agrícola con capacidad de almacenamiento variable. Ingeniería Hidráulica en México, 18(3), 81-93.

Fuentes, C., Namuche, R., Rendón, L., Patrón, R., Palacios, O., Brambila, F., \& González, A. (1997). Solución de la ecuación de Boussinesq del régimen transitorio en el drenaje agrícola bajo condiciones de radiación: El caso del Valle del Carrizo, Sinaloa. Memorias del VII Congreso Nacional de Irrigación, Hermosillo, Sonora, del 22 al 27 de octubre.

Fuentes, C., Brambila, F., Vauclin, M., Parlange, J.-Y., \& Haverkamp, R. (2001). Modelación fractal de la conductividad hidráulica de los suelos no saturados. Ingeniería Hidráulica en México, 16(2), 119137.

Fuentes, C., Zavala, M., \& Saucedo, H. (2009). Relationship between the storage coefficient and the soil-water retention curve in subsurface agricultural drainage systems: Water table drawdown. Journal of Irrigation and Drainage Engineering, ASCE, 135(3), 279-285, DOI: 10.1061/(ASCE)0733-9437(2009)135:3(279) 
Tecnología y

Ciencias đagua
2020, Instituto Mexicano de Tecnología del Agua

Open Access bajo la licencia CC BY-NC-SA 4.0

(https://creativecommons.org/licenses/by-nc-sa/4.0/)

Hooghoudt, S. (1940). Bijdragen tot de Kennis van eenige Natuurkundige Grootheden van de Grond. Verslagen and Landbouwkundige Onderzoekingen, 46(14), 515-707.

Mandelbrot, B. B. (1982). The fractal geometry of nature. New York, USA: W.H. Freeman and Company.

Nemes, A., Schaap, M. G., Leij, F. J., \& Wösten, J. H. M. (2001). Description of the unsaturated soil hydraulic database UNSODA version 2.0. Journal of Hydrology, 251(3-4), 151-162, DOI: 10.1016/S0022-1694(01)00465-6

Noor, A. K., \& Peters, J. M. (1987). Preconditioned conjugate gradient technique for the analysis of symmetric anisotropic structures. International Journal for Numerical Methods in Engineering, 24(11), 2057-2070, DOI: 10.1002/nme.1620241104

Ritzema, H. P. (2006). Drainage principles and applications. Wageningen, Netherlands: Alterra-ILRI.

Van Genuchten, M. Th. (1980). A close-form equation for predicting the hydraulic conductivity of unsaturated soils. Soil Science Society of American Journal, 44(5), 892-898. DOI: $10.2136 /$ sssaj1980.03615995004400050002x

Zavala, M., Fuentes, C., \& Saucedo, H. (2004). Radiación fractal en la ecuación de Boussinesq del drenaje agrícola. Ingeniería hidráulica en México, 19(3), 103-11.

Zavala, M., Fuentes, C., \& Saucedo, H. (2007). Non-linear radiation in the Boussinesq equation of the agricultural drainage. Journal of 
Teçnología y

Ciencias $₫$ Agua
2020, Instituto Mexicano de Tecnología del Agua

Open Access bajo la licencia CC BY-NC-SA 4.0

(https://creativecommons.org/licenses/by-nc-sa/4.0/)

Hydrology, $332(3-4)$,

374-380.

DOI: 10.1016/j.jhydrol.2006.07.009

Zavala, M., Saucedo, H., \& Fuentes, C. (2014). Programa de cómputo para analizar la dinámica del agua en sistemas de drenaje agrícola subterráneo. Agrociencia, 48(1), 71-85.

Zavala, M., Saucedo, H., \& Fuentes, C. (2018). Modelos analíticos fractales para las propiedades hidráulicas de suelos no saturados. Agrociencia, 52(8), 1059-1070.

Zienkiewicz, O. C., Taylor, R. L., \& Zhu, J. Z. (2013). The finite element method: Its basis and fundamentals. Amsterdam, The Netherlands: Elsevier. 